\title{
Hovering Localization and Power Allocation for UAV assisted DF Relaying Ad Hoc Network
}

\author{
Anirudh Agarwal* and Deepak Mishra ${ }^{\dagger}$ \\ ${ }^{*}$ Department of ECE, The LNM Institute of Information Technology, Jaipur, India \\ ${ }^{\dagger}$ School of Electrical Engineering and Telecommunications, University of New South Wales, Sydney, Australia
}

\begin{abstract}
An ad-hoc network using unmanned aerial vehicle (UAV) as relay, has been gaining significant attention especially for communication between far apart ground terminals during emergency conditions due to agility and resilience requirements. However, UAV hovering localization (HL) and power allocation (PA) are the key issues in such relay based flying ad hoc networks (FANETs). In this work, we propose a framework to jointly optimize the placement of rotary-wing UAV hovering and PA for maximization of network throughput in a three node decodeand-forward (DF) FANET. Specifically, we develop three different optimization schemes, (i) individual UAV HL optimization with a fixed allocated power, (ii) individual PA optimization for a fixed UAV HL, (iii) jointly optimal UAV HL and PA. For every optimization problem, the underlying convexity is proved and the global optimal solutions have been obtained. Further, we provide novel analysis by utilizing the characteristics of sigmoidal function thereby obtaining the closed-form and semi-closed-form expressions respectively for the globally-optimal solutions for individual and joint optimization schemes. The analytical results are numerically validated and various optimal design insights are discussed. It has been found that the proposed joint optimal scheme shows an average performance enhancement of $52 \%$ over benchmark scheme.
\end{abstract}

Index Terms-Ad hoc network, UAV, relay placement, hovering localization, power allocation, network throughput maximization.

\section{INTRODUCTION}

With the unprecedented advancement in $5 \mathrm{G}$ radio access technologies, the demand for ultra-high reliability, availability and adaptability of a wireless network is alarmingly increasing. In mission critical scenarios, such as public safety, disaster management or uninterrupted surveillance, the communication may be severely disrupted, where a sudden cost-effective solution is of utmost necessity. Flying ad hoc networks (FANETs), with unmanned aerial vehicles (UAVs) as relays, can provide seamless connectivity between the terrestrial users as a temporary recovery option in such situations [1]. Moreover, cooperative relaying using UAVs enhances the network coverage, end-to-end capacity and diversity gains [2]. However, few challenges like UAV hovering localization (HL) and transmit power allocation (PA) do exist in FANETs, and are required to be jointly optimized.

\section{A. State-of-the-Art}

The notion of UAV assisted cooperative communication has been well demonstrated in the existing literature [3]-[6]. In [7], UAV altitude was optimized using numerical simulations, considering Nakagami-m fading channel. In contrast, optimal

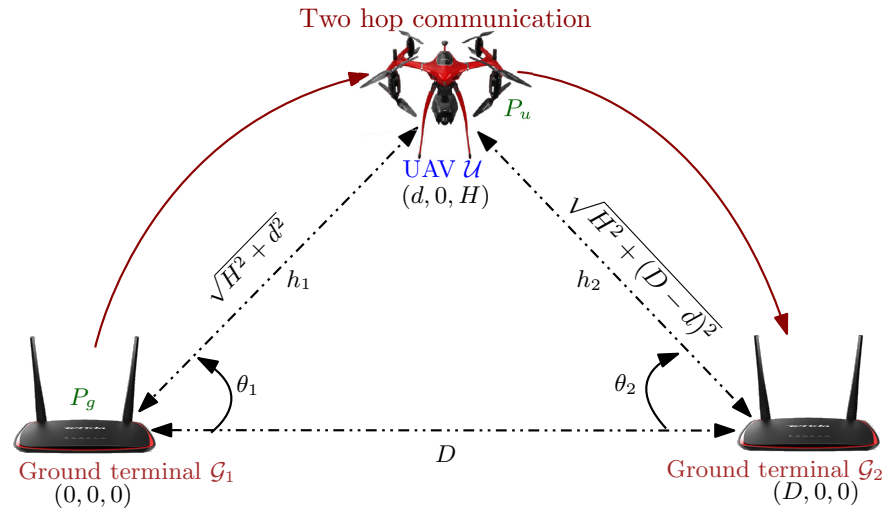

Fig. 1: Three-node $\left(\mathcal{G}_{1}, \mathcal{U}, \mathcal{G}_{2}\right)$ UAV relay-based network topology.

UAV HL was studied using deep learning approaches in [8], but neither the model was realistic nor the solutions were globally-optimal.

Few works also investigated the problem of optimal UAV HL and PA for throughput maximization. Specifically, in [9], an optimum UAV relay placement scheme was proposed while maximizing the UAV achievable rate. Further, authors in [10] maximized system throughput for a UAV-based mobile relaying station thereby optimizing the transmit PA and relay trajectory. Recently, [11] focused on optimal node placement and resource allocation for a UAV relaying network. However, these works have not considered non-line-of-sight (NLOS) component in the path loss model between any two communicating nodes. Alzenad et al. [12] did incorporate the line-of-sight (LOS) and NLOS components in their path loss model, and obtained optimal 3D placement of a UAV, but they considered UAV as an aerial base station [13].

\section{B. Motivation and Contribution}

Most of the existing works uniquely deal with the problem of either optimizing UAV HL or PA. Additionally, they consider only the LOS component in their path loss model. Few works did include the NLOS component [13], but the discussion on global optimality of the solutions has not yet been covered in the current literature. However, simultaneous analysis of optimal UAV HL and PA may drastically reduce the occasions of interrupted quality of service, thereby significantly improving the performance of FANETs. Therefore, to wholly exploit the benefits of a UAV-based aerial communication, we investigate the joint optimization of UAV HL and PA 
under varying real-world constraints. In particular, we study the performance of two-hop UAV assisted DF relaying FANET for maximizing the network throughput. Three optimization schemes have been proposed, viz. 1) Optimal HL: as there is no central-controller for allocating transmit power to ground terminals, so UAV relay is to be optimally positioned. 2) Optimal PA: due to variability factors in case of natural calamities like tsunamis/ cyclones, the hovering UAV may not be properly localized, and hence transmit PA optimization is required. 3) Joint optimal HL and PA: the merits of joint optimization offer enhanced overall network performance.

For practical applications, the proposed framework can be very beneficial to several stakeholders including public safety agencies, disaster management rescue bodies, defence organizations, where the communication between the terrestrial users is generally hampered.

To the best of our knowledge, this is the first work which investigates the joint optimization of UAV HL and PA in a $D F$ relaying ad hoc network considering both $L O S$ and NLOS components in the path loss model between the communicating nodes. The key contributions of our work are five-fold:

- The unique system model is proposed in Section II, in which the problem of joint optimization of UAV HL and PA is solved while considering both LOS and NLOS components in the path loss model.

- Convexity of the three optimization problems is proved in Section III.

- Global optimal solutions of HL and PA are obtained in Section IV, both individually and jointly.

- Closed-form expressions are provided for optimal PA, and HL in three specific UAV hovering scenarios.

- In Section V, we provide key optimal design insights along with the improved performance of the proposed schemes over benchmark, followed by conclusion in Section VI.

\section{Network Topology And Channel Model}

As presented in Fig. 1, we consider a three-node wireless ad hoc network in a temporary emergency situation, where a Cartesian coordinate system is used for node localization. The direct link between two ground terminals $\mathcal{G}_{1}$ and $\mathcal{G}_{2}$ located respectively at $(0,0,0)$ and $(D, 0,0)$ is negligible due to blockage. A fixed rotary-wing UAV $\mathcal{U}$ hovering at a height of $H \mathrm{~m}$ is thus employed at $(d, 0, H)$ as relay for assisting the communication between $\mathcal{G}_{1}$ and $\mathcal{G}_{2}$ using DF relaying strategy. Without loss of generality, we assume $\mathcal{G}_{1}$ as source node with transmit power $P_{g}$ and $\mathcal{G}_{2}$ as destination node communicating cooperatively via $\mathcal{U}$ with transmit power $P_{u}$. For effective utilization of energy resources, a common power budget $P_{\max }$ is considered such that $P_{\max }=P_{g}+P_{u}$. Further, we neglect the effect of small scale fading in the airto-ground (or ground-to-air) channel modeling as indicated in [1], and consider both LOS as well as NLOS components along with their separate probabilities of occurrence in path loss between communicating nodes [14]. However, in order to better highlight the novelty of the proposed model, we provide the channel power gain expression for two models, i.e. existing and proposed.

\section{A. Existing Model}

If the effect of only LOS component is included in the path loss model, the channel power gain is given by,

$$
\left|h_{i}\right|^{2}=\left|X_{i}\right|^{-n} \forall i \in\{1,2\},
$$

where $i=\{1,2\}$ respectively denote the link between $\mathcal{G}_{1}$ and $\mathcal{U}$, and the link between $\mathcal{U}$ and $\mathcal{G}_{2},\left|X_{1}\right| \triangleq \sqrt{H^{2}+d^{2}}$ is the distance between $\mathcal{G}_{1}$ and $\mathcal{U},\left|X_{2}\right| \triangleq \sqrt{H^{2}+(D-d)^{2}}$ is the distance between $\mathcal{U}$ and $\mathcal{G}_{2}, n$ is the path loss exponent.

\section{B. Proposed model}

Here, we incorporate the effect of LOS as well as NLOS component in the path loss model, thereby solving the problem of joint optimization of HL and PA. Consequently, the channel power gain is modified to,

$$
\left|h_{i}\right|^{2}=\left|X_{i}\right|^{-n} P R_{L_{i}}+\eta_{i}\left|X_{i}\right|^{-n} P R_{N L_{i}} \forall i \in\{1,2\},
$$

where $P R_{L_{i}}$ and $P R_{N L_{i}} \triangleq\left(1-P R_{L_{i}}\right)$ are the LOS and NLOS probabilities of occurrence corresponding to the $i^{\text {th }}$ communicating link, and $\eta_{i}$ is the $i^{t h}$ link attenuation factor due to NLOS connection. The LOS probability $P R_{L_{i}}$ is defined as [1],

$$
P R_{L_{i}}=\frac{1}{1+C_{i} \exp \left(-B_{i}\left(\theta_{i}-C_{i}\right)\right)} \forall i \in\{1,2\},
$$

where $\theta_{i}=\frac{180}{\pi} \sin ^{-1}\left(\frac{H}{\left|X_{i}\right|}\right)$ is the elevation angle of $\mathcal{U}$ with respect to the $i^{\text {th }}$ channel link.

Now, as the direct link between $\mathcal{G}_{1}$ and $\mathcal{G}_{2}$ is not available, the end-to-end SNR is restricted by weaker of SNRs of $\mathcal{G}_{1}$ to- $\mathcal{U}$ link $\gamma_{1} \triangleq\left|h_{1}\right|^{2} \frac{P_{g}}{\sigma^{2}}$ and $\mathcal{U}$-to- $\mathcal{G}_{2}$ link $\gamma_{2} \triangleq\left|h_{2}\right|^{2} \frac{P_{u}}{\sigma^{2}}$. Therefore, the network throughput $R_{\mathcal{G}_{1} \mathcal{G}_{2}}$ is defined as,

$$
R_{\mathcal{G}_{1} \mathcal{G}_{2}}=\log _{2}\left(1+\min \left\{\gamma_{1}, \gamma_{2}\right\}\right) \text {. }
$$

\section{OPTIMIZATION FRAMEWORK}

In this section, we present the optimization formulation, followed by the proof of convexity of optimization problem.

\section{A. Mathematical Formulation}

In order to maximize $R_{\mathcal{G}_{1} \mathcal{G}_{2}}$ subject to constraints on $d, H$ and $P_{g}$, following optimization problem has to be solved:

(P0): $\underset{d, P_{g}}{\operatorname{maximize}} R_{\mathcal{G}_{1} \mathcal{G}_{2}}$, subject to

$$
C 1: d \leq D, \quad C 2: H>H_{\min }, \quad C 3: P_{g}<P_{\max } .
$$

where $H_{\min }$ is the minimum allowable altitude of UAV.

As $\log ($.$) is a monotonically increasing function, so max-$ imizing the antilogarithm of $R_{\mathcal{G}_{1} \mathcal{G}_{2}}$, denoted as $\widehat{R}_{\mathcal{G}_{1} \mathcal{G}_{2}}=$ $\min \left\{\gamma_{1}, \gamma_{2}\right\}$ subject to constraints $C 1-C 3$, is equivalent to problem $(P 0)$. Therefore, the joint optimization problem of finding optimal PA $P_{g}^{*}$ and optimal $\mathrm{HL} d^{*}$ to maximize $\widehat{R}_{\mathcal{G}_{1} \mathcal{G}_{2}}$ subject to maximum UAV HL, minimum UAV altitude and total power constraints $(C 1-C 3)$ is given as:

$$
\text { (P1): } \underset{d, P_{g}}{\operatorname{maximize}} \widehat{R}_{\mathcal{G}_{1} \mathcal{G}_{2}} \text {, subject to } C 1, C 2, C 3 \text {. }
$$


Keeping boundary constraints $(C 1-C 3)$ implicit, the Lagrangian of $(P 1)$ is given by:

$$
\begin{aligned}
\mathcal{L}\left(d, P_{g}, \widehat{R}_{\mathcal{G}_{1} \mathcal{G}_{2}}, \lambda_{1}, \lambda_{2}\right) & =\widehat{R}_{\mathcal{G}_{1} \mathcal{G}_{2}}-\lambda_{1}\left(\widehat{R}_{\mathcal{G}_{1} \mathcal{G}_{2}}-\gamma_{1}\right) \\
& -\lambda_{2}\left(\widehat{R}_{\mathcal{G}_{1} \mathcal{G}_{2}}-\gamma_{2}\right),
\end{aligned}
$$

where $\lambda_{1}, \lambda_{2} \geq 0$ are the associated Lagrange multipliers.

\section{B. Proof for Convexity of $(P 1)$}

Here we provide Lemmas 1, 2 and 3, which together prove the convexity of $(P 1)$ [15].

Lemma 1: The objective $\widehat{R}_{\mathcal{G}_{1} \mathcal{G}_{2}}$ is a concave function of $d$.

Proof: Please refer to Appendix A.

Lemma 2: $\widehat{R}_{\mathcal{G}_{1} \mathcal{G}_{2}}$ is strictly pseudoconcave in $d$ and $P_{g}$.

Proof: $\widehat{R}_{\mathcal{G}_{1} \mathcal{G}_{2}}$ is a concave function of $d$ from Lemma 1 , and is linear in $P_{g}$, hence $\widehat{R}_{\mathcal{G}_{1} \mathcal{G}_{2}}$ proves to be strictly pseudoconcave function of $d$ and $P_{g}$ [16, Table 5.2].

Lemma 3: Constraints $C 1, C 2, C 3$ form a convex set.

Proof: Since constraints $C 1, C 2, C 3$ are linear in $d$ as well as $P_{g}$, therefore they form a convex set.

From Lemmas 1, 2 and 3, $(P 1)$ is a generalized convex optimization problem in $d$ and $P_{g}$.

\section{Globally-Optimal HL and PA Solutions}

As $(P 1)$ is a generalized convex optimization problem in $d$ and $P_{g}$ from Section III. B, the underlying Karush-KuhnTucker (KKT) point $\left(d^{*}, P_{g}^{*}, \lambda_{1}^{*}, \lambda_{2}^{*}\right)$ provides the joint globally-optimal solution of $(P 1)$ [17, Theorem 4.3.8]. The corresponding KKT conditions are,

$$
\begin{gathered}
\frac{\partial \mathcal{L}}{\partial d} \triangleq \lambda_{1} \frac{\partial \gamma_{1}}{\partial d}+\lambda_{2} \frac{\partial \gamma_{2}}{\partial d}=0, \\
\frac{\partial \mathcal{L}}{\partial P_{g}} \triangleq \lambda_{1}\left(\frac{\left|h_{1}\right|^{2}}{\sigma^{2}}\right)-\lambda_{2}\left(\frac{\left|h_{2}\right|^{2}}{\sigma^{2}}\right)=0, \\
\frac{\partial \mathcal{L}}{\partial \widehat{R}_{\mathcal{G}_{1} \mathcal{G}_{2}}} \triangleq 1-\lambda_{1}-\lambda_{2}=0 .
\end{gathered}
$$

Since both $\gamma_{1}$ as well as $\gamma_{2}$ are monotonic in each of the variables $d$ and $P_{g}$, and $\lambda_{1}, \lambda_{2} \geq 0$, thus (8a), (8b), (8c) can simultaneously hold true only when,

$$
\frac{\partial \gamma_{1}}{\partial d}=\frac{\partial \gamma_{2}}{\partial d}
$$

Therefore, from the above discussion, the global optimal solution of $\left(P_{1}\right)$ in $d$ and $P_{g}$ will satisfy:

$$
\gamma_{1}=\gamma_{2}
$$

We next provide three optimization schemes to maximize $\widehat{R}_{\mathcal{G}_{1} \mathcal{G}_{2}}$, thereby obtaining the semi-closed-form optimal solutions.

\section{A. Fully Adaptive Scheme: Joint Optimization of PA and HL}

After solving (10) in $P_{g}^{*}$ (as a function of $d$ ), we get $P_{g}^{*}=\frac{\left|h_{2}\right|^{2} P_{\max }}{\left|h_{1}\right|^{2}+\left|h_{2}\right|^{2}}$. Thus, the problem $(P 1)$ can be reformulated explicitly as a function of $d$ as follows:

$$
\begin{gathered}
(P 2): \underset{d}{\operatorname{maximize}} \frac{\left|h_{1}\right|^{2}\left|h_{2}\right|^{2}}{\left|h_{1}\right|^{2}+\left|h_{2}\right|^{2}} \frac{P_{\max }}{\sigma^{2}} \\
\text { subject to } C 1, C 2, C 3 .
\end{gathered}
$$

Since $\widehat{R}_{\mathcal{G}_{1} \mathcal{G}_{2}}$ is a concave function of $d$ from Lemma 1 , therefore, the objective in $(P 2)$ is also a concave function of $d$ [15] which leads to convexity of the problem $(P 2)$ due to linearity of constraints $C 1, C 2, C 3$ in $d$. Consequently, the following condition holds true for maxima:

$$
\frac{\partial}{\partial d}\left(\frac{\left|h_{1}\right|^{2}\left|h_{2}\right|^{2}}{\left|h_{1}\right|^{2}+\left|h_{2}\right|^{2}} \frac{P_{\max }}{\sigma^{2}}\right)=0 .
$$

Simplifying (12) further results in,

$$
\left|h_{1}\right|^{4} \frac{\partial\left|h_{2}\right|^{2}}{\partial d}+\left|h_{2}\right|^{4} \frac{\partial\left|h_{1}\right|^{2}}{\partial d}=0
$$

Finally, considering free-space path loss (i.e. $n=2$ ), a univariable eq. in $d$ from (13) is obtained as,

$$
\begin{aligned}
& \alpha_{1}^{2}\left[\mu_{2}\left(\alpha_{2}-\eta_{2}\right)\left(1-\alpha_{2}\right)+2(D-d) \alpha_{2}\right] \\
& -\alpha_{2}^{2}\left[\mu_{1}\left(\alpha_{1}-\eta_{1}\right)\left(1-\alpha_{1}\right)-2 d \alpha_{1}\right]=0,
\end{aligned}
$$

where $\alpha_{i} \triangleq\left(1-\eta_{i}\right) P R_{L_{i}}+\eta_{i}, \mu_{i}=\frac{180}{\pi} \frac{B H}{1-\eta_{i}} \forall i \in\{1,2\}$.

We solve (14) for specific UAV applications $\left(\theta_{1}=\theta_{2} \triangleq \Theta\right)$ to obtain optimal $d^{*}$ as,

$$
d^{*}=\frac{\zeta_{2}-\zeta_{1}-2 D \alpha_{1}^{2} \alpha_{2}}{2 \alpha_{1} \alpha_{2}\left(\alpha_{2}-\alpha_{1}\right)},
$$

where $\zeta_{i}=\alpha_{i}^{2}\left[\mu_{j}\left(\alpha_{j}-\eta_{j}\right)\left(1-\alpha_{j}\right)\right] \forall i, j \in\{1,2\}, i \neq j$, and $\alpha_{i}=\alpha_{i} \forall \theta_{i}=\Theta, i \in\{1,2\}$. For $\Theta$, we have considered following three special UAV hovering scenarios:

1) UAV Hovering at Large Height: $H_{\min } \gg D$, therefore $\theta_{1}=\theta_{2} \triangleq \Theta \approx 90^{\circ}$.

2) UAV Hovering at Small Height: $H_{\text {min }} \ll d_{\text {min }}$, hence $\theta_{1}=\theta_{2} \triangleq \Theta \approx 0^{\circ}$.

3) UAV Hovering Equidistant from $\mathcal{G}_{1}$ and $\mathcal{G}_{2}: d=\frac{D}{2}$, consequently $\theta_{1}=\theta_{2} \triangleq \Theta \approx \frac{180}{\pi} \tan ^{-1}\left(\frac{2 H}{D}\right)$.

However for a generalized path loss scenario, we solve (P2) in $d^{*}$ with the help of Golden Section Line Search (GSLS) method [18]. The number of computations $N_{C}^{G S}$ in GSLS algorithm is given by $N_{C}^{G S}=\left[2-2.08 \ln \left(\frac{\xi}{d_{U}^{*}-d_{L}^{*}}\right)\right]$, where $\xi$ is the acceptable tolerance, $d_{L}^{*}$ and $d_{U}^{*}$ are respectively the lower and upper bound on $d^{*}$.

\section{B. First Semi-adaptive Scheme: Optimal HL for a Fixed PA}

For a predetermined PA $\left(P_{g}, P_{u}\right)$, the problem of optimal HL (OHL) that maximizes $\widehat{R}_{\mathcal{G}_{1} \mathcal{G}_{2}}$ subject to constraint $C 1$ is given by:

$$
\text { (P3): } \underset{d}{\operatorname{maximize}} \widehat{R}_{\mathcal{G}_{1} \mathcal{G}_{2}} \text {, subject to } C 1 \text {. }
$$

As $\widehat{R}_{\mathcal{G}_{1} \mathcal{G}_{2}}$ is a concave function of $d$ from Lemma 1 , and constraint $C 1$ is linear in $d$, thus (P3) is convex, and from ( $8 \mathrm{a})$, (8c), the global optimum solution can be obtained by solving (10) in $d$, because $\lambda_{i} \geq 0$ and $\gamma_{i}$ are monotonic functions of $d$. Therefore, (10) can now be solved in $d$ to obtain OHL $d_{a}$ numerically for a fixed $P_{g}$ via GSLS method.

Furthermore, we utilize few key characteristics of sigmoidal function thereby proposing two approximation approaches to obtain the value of OHL using (10):

1) Step Approximation: As the LOS probability is characterized by a sigmoidal function, $P R_{L_{i}}=\frac{1}{1+C_{i} \exp \left(-B_{i}\left(\theta_{i}-C_{i}\right)\right)}$ $\forall i \in\{1,2\}$, so for high values of $B_{i}$, i.e., $B_{i}>1$, 
$P R_{L_{i}} \in\{0,1\}$. Consider the case when $P R_{L_{1}}=1$ and $P R_{L_{2}}=0$, therefore, from (10) we have,

$$
\frac{P_{g}}{\left|X_{1}\right|^{n}}=\frac{\eta_{2}\left(P_{\max }-P_{g}\right)}{\left|X_{2}\right|^{n}} \text {. }
$$

After few mathematical arrangements in (17), the closed-form solution in OHL $d_{s a}$ can be obtained as,

$$
d_{s a}=\mathcal{D}-\sqrt{\mathcal{D}^{2} \rho-H^{2}}
$$

where $\mathcal{D} \triangleq \frac{D}{1-\rho}, \rho=\left[\eta_{2}\left(\frac{P_{\max }}{P_{g}}-1\right)\right]^{\frac{2}{n}}$.

2) Linear Approximation: If the value of $B_{i} \forall i \in\{1,2\}$ is small enough $\left(B_{i}<0.01\right)$ to approximately linearize the sigmoidal function, i.e., $P R_{L_{i}}$ is linear in $\theta_{i}$, then $P R_{L_{i}}$ (as a function of $\theta_{i}$ ) is given by:

$$
P R_{L_{i}}\left(\theta_{i}\right)=m_{i} \theta_{i}+k_{i},
$$

where $m_{i}=\frac{\partial P R_{L_{i}}\left(0^{\circ}\right)}{\partial \theta_{i}}, k_{i}=P R_{L_{i}}\left(0^{\circ}\right) \forall i \in\{1,2\}$. Therefore, from (10) and (19),

$$
\begin{aligned}
\left|\frac{X_{2}}{X_{1}}\right|^{n} & =\frac{\left(P_{\max }-P_{g}\right)\left\{\left(1-\eta_{2}\right)\left(m_{2} \theta_{2}+k_{2}\right)+\eta_{2}\right\}}{P_{g}\left\{\left(1-\eta_{1}\right)\left(m_{1} \theta_{1}+k_{1}\right)+\eta_{1}\right\}} \\
& \triangleq \varrho\left(\theta_{1}, \theta_{2}\right) .
\end{aligned}
$$

Let us initially assume $\theta_{i} \forall i \in\{1,2\}$ in (20) to be independent of $d$ present in $\left|X_{i}\right| \forall i \in\{1,2\}$ at the left-hand side of (20), then similar to (18),

$$
d=\mathfrak{D}-\sqrt{\mathfrak{D}^{2} \varrho\left(\theta_{1}, \theta_{2}\right)-H^{2}},
$$

where $\mathfrak{D} \triangleq \frac{D}{1-\varrho\left(\theta_{1}, \theta_{2}\right)}$.

Now, we obtain optimal OHL $d_{l a}$ by solving (21) in $d$ for three different UAV communication scenarios as proposed above in fully adaptive scheme:

$$
d_{l a i}=\mathfrak{D}_{i}-\sqrt{\mathfrak{D}_{i}^{2} \varrho\left(\phi_{i}, \phi_{i}\right)-H^{2}},
$$

where $\mathfrak{D}_{i} \triangleq \frac{D}{1-\varrho\left(\phi_{i}, \phi_{i}\right)}, \phi_{i} \in\left\{90^{\circ}, 0^{\circ}, \frac{180}{\pi} \tan ^{-1}\left(\frac{2 H}{D}\right)\right\}, i \in$ $\{1,2,3\}$.

\section{Second Semi-Adaptive Scheme: Optimal PA for a Fixed HL}

In this scheme, we study the optimal PA (OPA) problem for a given HL thereby maximizing $\widehat{R}_{\mathcal{G}_{1} \mathcal{G}_{2}}$ subject to total power constraint $C 3$, which leads to the formulation of the following optimization problem:

$$
\text { (P3): } \underset{P_{g}}{\operatorname{maximize}} \widehat{R}_{\mathcal{G}_{1} \mathcal{G}_{2}} \text {, subject to } C 3 \text {. }
$$

Since $\widehat{R}_{\mathcal{G}_{1} \mathcal{G}_{2}}$ is linear in $P_{g}$, and $\frac{\partial^{2} \widehat{R}_{\mathcal{G}_{1} \mathcal{G}_{2}}}{\partial P_{g}^{2}}=0$ which is a necessary condition for concavity of a function [15]. Moreover, constraint $C 3$ forms a convex set, hence $(P 4)$ is a convex optimization problem [15]. Consequently, from (8b) and (8c), the globally-optimal PA solution is the root of (10) in $P_{g}$.

For a fixed $d,(10)$ can be solved for joint optimal PA, $P_{g a}$ as follows:

$$
P_{g a} \triangleq \frac{\alpha_{2} \beta_{1}}{\alpha_{1} \beta_{2}+\alpha_{2} \beta_{1}} P_{\max },
$$

where $\beta_{i} \triangleq\left|X_{i}\right|^{n}, \forall i \in\{1,2\}$.

\section{Results and Performance Evaluation}

In this section, the key numerical insights of the two individual OHL and OPA schemes along with joint optimization

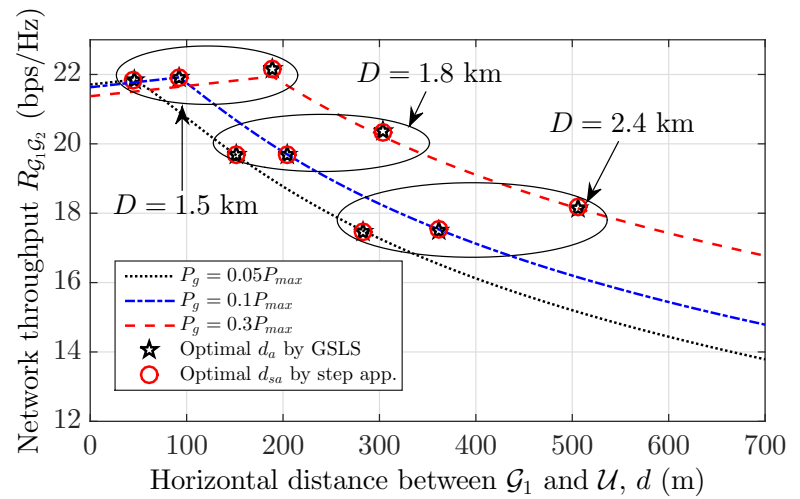

Fig. 2: Impact of $D$ on variation of $R_{\mathcal{G}_{1} \mathcal{G}_{2}}$ and OHL at high $B$.

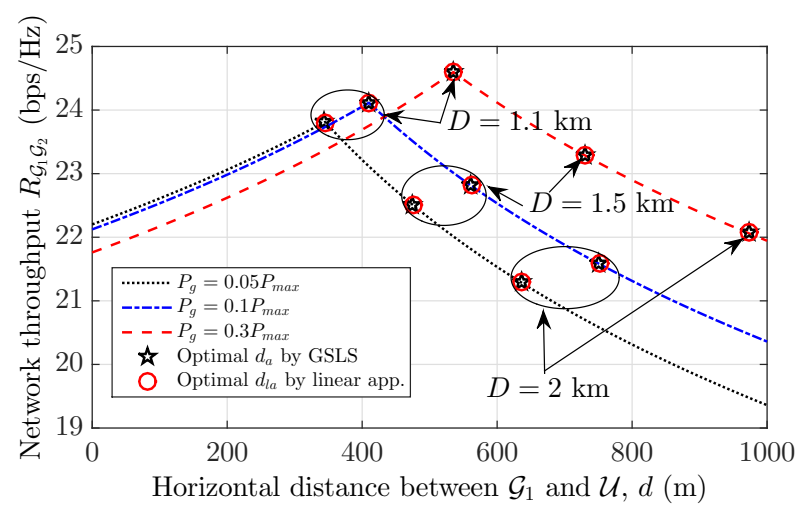

Fig. 3: Influence of $D$ on variation of $R_{\mathcal{G}_{1} \mathcal{G}_{2}}$ and OHL at low $B$.

of HL and PA scheme have been discussed. Unless explicitly stated, we have considered, $P_{\max }=10 \mathrm{~W}, \sigma^{2}-100 \mathrm{dBm}$, $n=3, \eta_{1}=200, \eta_{2}=100$. The parameters for dense urban environment are assumed to be equal for two communicating links, i.e. $B_{1}=B_{2} \triangleq B=0.35$ and $C_{1}=C_{2} \triangleq C=5$. For comparison, we undertake a uniform fixed localization and PA scheme as the benchmark with HL as $d=\frac{D}{2}$ and PA as $P_{g}=P_{u}=\frac{P_{\max }}{2}$.

\section{A. Performance Evaluation of OHL Scheme}

For validating the proposed scheme to obtain OHL for a fixed PA, we plot the variation of $R_{\mathcal{G}_{1} \mathcal{G}_{2}}$ as a function of $d$ for three fixed PAs at different $D$ in Figs. 2 and 3. At high value of environment parameter $B=5$, the OHL values obtained by using GSLS and step approximation (app.) are found to exactly match with the maximum $R_{\mathcal{G}_{1} \mathcal{G}_{2}}$ in Fig. 2. Similar behavior can be observed for linear approximation based OHL in Fig. 3 for low $B=0.01$. Moreover, as $D$ becomes large, $R_{\mathcal{G}_{1} \mathcal{G}_{2}}$ due to OHL is reduced because of increased path loss between both the communication links. However, this leads to increase in OHL which shifts $\mathcal{U}$ towards $\mathcal{G}_{2}$ to strengthen the weakened $\mathcal{U}-\mathcal{G}_{2}$ link.

\section{B. Performance Evaluation of OPA Scheme}

In Fig. 4, we vary $R_{\mathcal{G}_{1} \mathcal{G}_{2}}$ against $P_{g}$ for different values of $d$ to validate the global OPA solution as obtained from (23). Network throughput due to OPA is improved with source power $P_{g}$, because with high $P_{g}$, the $\mathcal{G}_{1}-\mathcal{U}$ link 


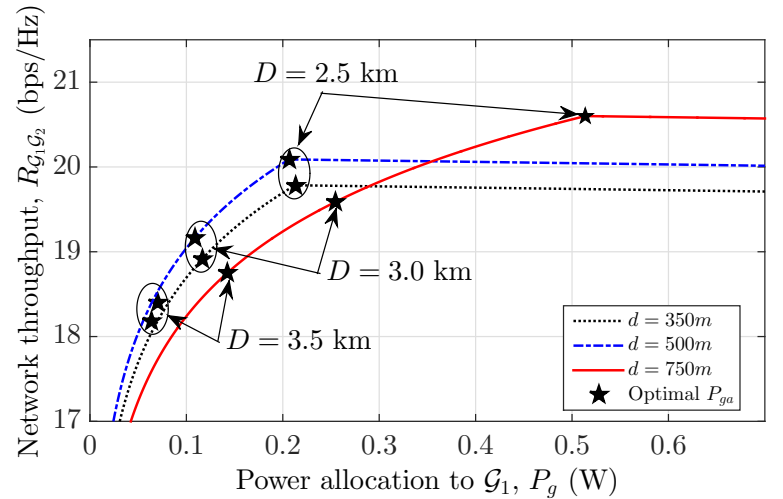

Fig. 4: Effect of $D$ on variation of $R_{\mathcal{G}_{1} \mathcal{G}_{2}}$ and OPA at different $d$.

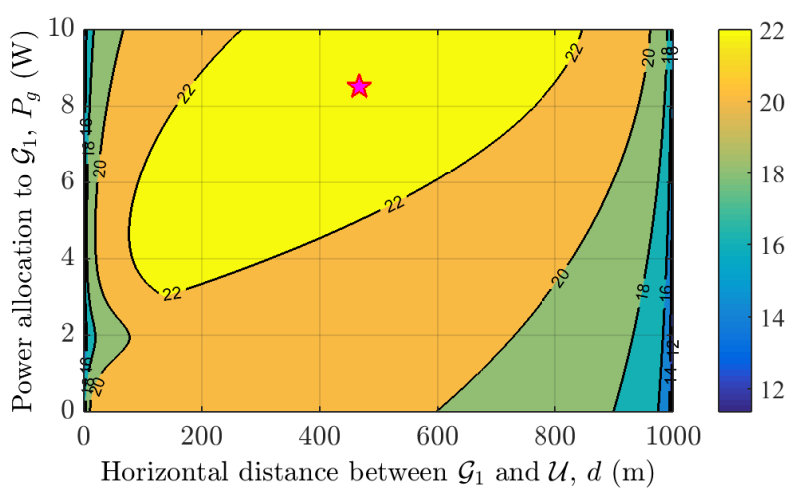

Fig. 5: Joint optimization of HL and PA and the maximized $R_{\mathcal{G}_{1} \mathcal{G}_{2}}$.

gets strengthened. However, further increase in $P_{g}$ pushes $\mathcal{U}$ towards $\mathcal{G}_{2}$ by which $\mathcal{U}-\mathcal{G}_{2}$ becomes vigorous, therefore $R_{\mathcal{G}_{1} \mathcal{G}_{2}}$ increases with high $d$ value. Further, for low values of $D$, both $\mathcal{G}_{1}-\mathcal{U}$ and $\mathcal{U}-\mathcal{G}_{2}$ links get sufficient strength with increase in elevation angle that leads to mitigated path loss, hence the overall throughput is enhanced.

\section{Performance Evaluation of Joint Optimization Scheme}

A contour plot has been provided in Fig. 5, showing the variation of $R_{\mathcal{G}_{1} \mathcal{G}_{2}}$ for different values of $d$ and $P_{g}$. Additionally, we plot the joint optimal HL and PA value with a magenta-colored pentagram. It can be noticed that the joint optimal point $\left(d^{*}, P_{g}^{*}\right)$ is obtained in the feasible region with a large source power $P_{g}$ and lower $d$ value. This is attributed to the fact that more $P_{g}$ and less $d$ respectively improves the SNR and reduces the path loss of the $\mathcal{G}_{1}-\mathcal{U}$ link, which leads to maximized network throughput. Moreover, if $P_{g}$ is drastically increased, then the maximum $R_{\mathcal{G}_{1} \mathcal{G}_{2}}$ due to joint optimal HL and PA is achieved at a higher value of $d^{*}$ because of the strengthened $\mathcal{U}-\mathcal{G}_{2}$ link. So, joint optimal $P_{g}^{*}$ and $d^{*}$ simultaneously govern the strength of $\mathcal{G}_{1}-\mathcal{U}$ and $\mathcal{U}-\mathcal{G}_{2}$ links.

\section{Impact of Environment Conditions on Optimal Design}

In Fig. 6, the joint OHL and joint OPA have been plotted as a function of $D$ for different values of environment parameter $B$. It is observed that in any environment, $d^{*}$ increases with $D$, because large $D$ leads to suppression in elevation angle of $\mathcal{U}$ from $\mathcal{G}_{1}$, which actually increases $d^{*}$ with stronger $\mathcal{U}-\mathcal{G}_{2}$

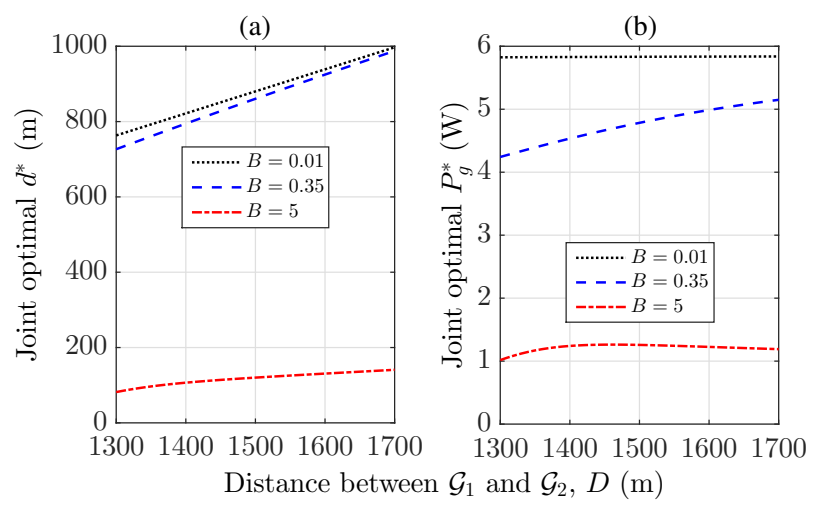

Fig. 6: Variation of joint optimal $d^{*}$ and $P_{g}^{*}$ with $D$ for different $B$.

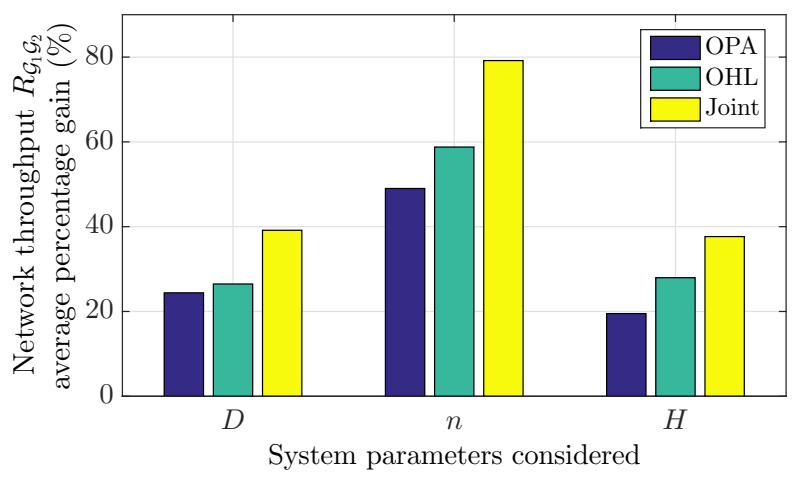

Fig. 7: Comparison of proposed schemes against benchmark.

link. Simultaneously, $P_{g}^{*}$ also gets increased in order to fully strengthen the weakened $\mathcal{G}_{1}-\mathcal{U}$ link when $D$ is more. So, similar behavior can be seen in low $B$ environment, as the path loss varies linearly with the elevation angle. On the other hand, in high $B$ environment, optimal $P_{g}^{*}$ is achieved at lower value, as less power is required due to the nullified effect of NLOS component in $\mathcal{G}_{1}-\mathcal{U}$ link. But, in order to increase the SNR of the $\mathcal{G}_{1}-\mathcal{U}$ link, optimal $d^{*}$ value is also low, thereby further reducing the path loss.

\section{E. Performance Comparison and Achievable Gain}

For comparative analysis, we plot the average $R_{\mathcal{G}_{1} \mathcal{G}_{2}}$ percentage gain achieved using the proposed OPA, OHL and joint optimization schemes over the benchmark scheme for different system parameters such as $D, n, H$ in Fig. 7. Clearly, the proposed schemes outperform the benchmark for any parameter with a mean improvement of almost $52 \%$ in case of joint optimal scheme, because due to fixed allocation, the benchmark scheme fails to provide optimal value with respect to any geographical or environment change in the networking scenario.

\section{CONCLUSION}

This work investigated the problem of joint as well as individual optimization UAV HL and PA in a DF relaying based two-hop wireless ad hoc network. We successfully proved the convexity of three optimization problems and obtained the global optimal solutions. Furthermore, we provided various 
closed-form and semi-closed-form expressions of the solutions corresponding to step and linear approximations in case of individual optimal $\mathrm{HL}$, generalized scenario in case of individual PA, and some specific UAV hovering applications in case of joint optimal $\mathrm{HL}$ and PA. The results are numerically validated for all the obtained optimal solutions. Finally, a significant performance enhancement is observed by the proposed scheme over the benchmark.

\section{APPENDIX A}

\section{PROOF OF LEMMA 1}

In this appendix, we provide the proof of Lemma 1. Let us consider Consider $\left|h_{1}\right|^{2}$, which is the ratio of $\alpha_{1} \triangleq(1-$ $\left.\eta_{1}\right) P R_{L_{1}}+\eta_{1}$ and $\beta_{1} \triangleq\left|X_{1}\right|^{n}$. Clearly, $\alpha_{1}, \beta_{1}>0 \forall \eta_{1}>0$ and $0 \leq P R_{L_{1}} \leq 1$. Moreover,

$$
\begin{aligned}
\frac{\partial \alpha_{1}}{\partial d} \triangleq & {\left[\left(\eta_{1}-1\right) \frac{180}{\pi} \frac{H}{H^{2}+d^{2}} B_{1} P R_{L_{1}}\left(1-P R_{L_{1}}\right)\right]>0 } \\
& \forall \eta_{1}>1, B_{1}>0,
\end{aligned}
$$

$$
\begin{gathered}
\frac{\partial \beta_{1}}{\partial d} \triangleq n d\left(H^{2}+d^{2}\right)^{\frac{n-2}{2}}>0 \forall n>0, \\
\frac{\partial^{2} \alpha_{1}}{\partial d^{2}} \triangleq\left[( \eta _ { 1 } - 1 ) \frac { 1 8 0 } { \pi } \frac { H } { H ^ { 2 } + d ^ { 2 } } B _ { 1 } P R _ { L _ { 1 } } ^ { 2 } C _ { 1 } \left(B _ { 1 } H \left\{2 P R_{L_{1}}\right.\right.\right. \\
-1\}-2 d)]<0 \forall B_{1} H<2 d,
\end{gathered}
$$

$\frac{\partial^{2} \beta_{1}}{\partial d^{2}} \triangleq\left[n\left(H^{2}+d^{2}\right)^{\frac{n-4}{2}}\left\{H^{2}+(n-1) d^{2}\right\}\right]>0 \forall n>1$.

Therefore, from (A.1a)-(A.1d),

$$
\begin{aligned}
\frac{\partial^{2}\left|h_{1}\right|^{2}}{\partial d^{2}} & \triangleq \frac{1}{A_{2}^{4}}\left[A_{2}^{2}\left\{\beta_{1} \frac{\partial^{2} \alpha_{1}}{\partial d^{2}}-\alpha_{1} \frac{\partial^{2} \beta_{1}}{\partial d^{2}}\right\}\right. \\
& \left.-2 \beta_{1} \frac{\partial \beta_{1}}{\partial d}\left\{\beta_{1} \frac{\partial \alpha_{1}}{\partial d}-\alpha_{1} \frac{\partial \beta_{1}}{\partial d}\right\}\right]<0,
\end{aligned}
$$

which implies that $\left|h_{1}\right|^{2}$ is strictly concave in $d$ [15].

Similarly, $\left|h_{2}\right|^{2} \triangleq \frac{\alpha_{2}}{\beta_{2}}$, where $\alpha_{2} \triangleq\left(1-\eta_{2}\right) P R_{L_{2}}+\eta_{2}$ and $\beta_{2} \triangleq\left|X_{2}\right|^{n}$. It can be seen that, $\alpha_{2}, \beta_{2}>0 \forall \eta_{2}>0$ and $0 \leq P R_{L_{2}} \leq 1$. Further,

$$
\begin{aligned}
\frac{\partial \alpha_{2}}{\partial d} & \triangleq\left[\left(1-\eta_{2}\right) \frac{180}{\pi} \frac{H}{H^{2}+(D-d)^{2}} B_{2} P R_{L_{2}}\left(1-P R_{L_{2}}\right)\right] \\
& <0 \forall \eta_{2}>1, B_{2}>0,
\end{aligned}
$$

$$
\begin{aligned}
& \frac{\partial \beta_{2}}{\partial d} \triangleq\left.n(d-D)\left(H^{2}+(D-d)^{2}\right)^{\frac{n-2}{2}}<0 \forall n>0, \text { (A.3 }\right) \\
& \frac{\partial^{2} \alpha_{2}}{\partial d^{2}} \triangleq\left[( \eta _ { 2 } - 1 ) \frac { 1 8 0 } { \pi } \frac { H } { H ^ { 2 } + ( D - d ) ^ { 2 } } B _ { 2 } P _ { L _ { 2 } } ^ { 2 } C _ { 2 } \left(B_{2} H\right.\right. \\
& \\
&\left.\left.\times\left\{2 P R_{L_{2}}-1\right\}-2 d\right)\right]<0 \forall B_{2} H<2(D-d),
\end{aligned}
$$

$$
\begin{aligned}
\frac{\partial^{2} \beta_{2}}{\partial d^{2}} & \triangleq\left[n ( H ^ { 2 } + ( D - d ) ^ { 2 } ) ^ { \frac { n - 4 } { 2 } } \left\{H^{2}+(n-1)\right.\right. \\
& \left.\left.\times(D-d)^{2}\right\}\right]>0 \forall n>1 .
\end{aligned}
$$

As a result from (A.3a)-(A.3d),

$$
\begin{aligned}
\frac{\partial^{2}\left|h_{2}\right|^{2}}{\partial d^{2}} & \triangleq \frac{1}{A_{4}^{4}}\left[A_{4}^{2}\left\{\beta_{2} \frac{\partial^{2} \alpha_{2}}{\partial d^{2}}-\alpha_{2} \frac{\partial^{2} \beta_{2}}{\partial d^{2}}\right\}\right. \\
& \left.-2 \beta_{2} \frac{\partial \beta_{2}}{\partial d}\left\{\beta_{2} \frac{\partial \alpha_{2}}{\partial d}-\alpha_{2} \frac{\partial \beta_{2}}{\partial d}\right\}\right]<0,
\end{aligned}
$$

and hence $\left|h_{2}\right|^{2}$ is strictly concave in $d$.

As both $\left|h_{1}\right|^{2}$ and $\left|h_{2}\right|^{2}$ are concave functions of $d$, so the minimum of two concave functions is also a concave function [15], thus $\widehat{R}_{\mathcal{G}_{1} \mathcal{G}_{2}}$ is a concave function of $d$.

\section{REFERENCES}

[1] A. Al-Hourani, S. Kandeepan, and S. Lardner, "Optimal LAP altitude for maximum coverage," IEEE Wireless Commun. Lett., vol. 3, no. 6 , pp. 569-572, Dec. 2014.

[2] D. Mishra, S. De, S. Jana, S. Basagni, K. Chowdhury, and W. Heinzelman, "Smart RF energy harvesting communications: challenges and opportunities," IEEE Commun. Mag., vol. 53, no. 4, pp. 70-78, Apr. 2015.

[3] K. A.M, F. Hu, and S. Kumar, "Deep $Q$-learning-based node positioning for throughput-optimal communications in dynamic UAV swarm network," IEEE Trans. on Cognitive Communications and Networking, vol. 5, no. 3, pp. 554-566, Sep. 2019.

[4] F. Cheng, G. Gui, N. Zhao, Y. Chen, J. Tang, and H. Sari, "UAV-relayingassisted secure transmission with caching," IEEE Trans. on Commun. vol. 67, no. 5, pp. 3140-3153, May 2019.

[5] B. Ji, Y. Li, B. Zhou, C. Li, K. Song, and H. Wen, "Performance analysis of UAV relay assisted IoT communication network enhanced with energy harvesting," IEEE Access, vol. 7, pp. 38738-38 747, Mar. 2019.

[6] Y. Zeng, R. Zhang, and T. J. Lim, "Wireless communications with unmanned aerial vehicles: opportunities and challenges," IEEE Commun. Mag., vol. 54, no. 5, pp. 36-42, May 2016.

[7] Y. Chen, W. Feng, and G. Zheng, "Optimum placement of UAV as relays," IEEE Commun. Lett., vol. 22, no. 2, pp. 248-251, Feb. 2018.

[8] Y. Yayeh Munaye, H.-P. Lin, A. B. Adege, and G. B. Tarekegn, "UAV positioning for throughput maximization using deep learning approaches," Sensors, vol. 19, no. 12, p. 2775, 2019.

[9] F. Ono, H. Ochiai, and R. Miura, "A wireless relay network based on unmanned aircraft system with rate optimization," IEEE Trans. on Wireless Commun., vol. 15, no. 11, pp. 7699-7708, Nov. 2016.

[10] Y. Zeng, R. Zhang, and T. J. Lim, "Throughput maximization for UAVenabled mobile relaying systems," IEEE Trans. on Commun., vol. 64 no. 12, pp. 4983-4996, Dec. 2016.

[11] R. Fan, J. Cui, S. Jin, K. Yang, and J. An, "Optimal node placement and resource allocation for UAV relaying network," IEEE Commun. Lett., vol. 22, no. 4, pp. 808-811, Apr. 2018.

[12] M. Alzenad, A. El-Keyi, F. Lagum, and H. Yanikomeroglu, "3-d placement of an unmanned aerial vehicle base station (UAV-BS) for energyefficient maximal coverage," IEEE Wireless Commun. Lett., vol. 6, no. 4 pp. 434-437, Aug. 2017.

[13] P. Lohan and D. Mishra, "Utility-aware optimal resource allocation protocol for uav-assisted small cells with heterogeneous coverage demands," IEEE Trans. on Wireless Commun., vol. 19, no. 2, pp. 1221-1236, Feb 2020.

[14] A. Al-Hourani, S. Kandeepan, and A. Jamalipour, "Modeling air-toground path loss for low altitude platforms in urban environments," in 2014 IEEE GLOBECOM, Austin, Dec. 2014, pp. 2898-2904.

[15] S. Boyd and L. Vandenberghe, Convex optimization. Cambridge University Press, 2004.

[16] M. Avriel, W. E. Diewert, S. Schaible, and I. Zang, Generalized concavity. Siam, 2010, vol. 63.

[17] M. S. Bazaraa, H. D. Sherali, and C. M. Shetty, Nonlinear programming: theory and algorithms. John Wiley \& Sons, 2013.

[18] A. D. Belegundu and T. R. Chandrupatla, Optimization concepts and applications in engineering. Cambridge University Press, 2019. 